\title{
CLUSTER MERGER SHOCK CONSTRAINTS ON PARTICLE ACCELERATION AND NONTHERMAL PRESSURE IN THE INTRACLUSTER MEDIUM
}

\author{
Ehud NaKar, ${ }^{1}$ Miloš Milosavluević, ${ }^{2}$ and Daisuke Nagai ${ }^{1}$ \\ Received 2007 June 18; accepted 2007 October 2
}

\begin{abstract}
X-ray observations of galaxy cluster merger shocks can be used to constrain nonthermal processes in the intracluster medium (ICM). The presence of nonthermal pressure components in the ICM, as well as the shock acceleration of particles and their escape, all affect shock jump conditions in distinct ways. Therefore, these processes can be constrained using X-ray surface brightness and temperature maps of merger shock fronts. Here we use these observations to place constraints on particle acceleration efficiency in intermediate Mach number $(\mathcal{M} \approx 2-3)$ shocks and explore the potential to constrain the contribution of nonthermal components (e.g., cosmic rays, magnetic field, and turbulence) to ICM pressure in cluster outskirts. We model the hydrodynamic jump conditions in merger shocks discovered in the galaxy clusters $\mathrm{A} 520(\mathcal{M} \approx 2)$ and $1 \mathrm{E} 0657-56(\mathcal{M} \approx 3)$ using a multifluid model comprising a thermal plasma, a nonthermal plasma, and a magnetic field. Based on the published X-ray spectroscopic data alone, we find that the fractional contribution of cosmic rays accelerated in these shocks is $\$ 10 \%$ of the shock downstream pressure. Current observations do not constrain the fractional contribution of nonthermal components to the pressure of the undisturbed shock upstream. Future X-ray observations, however, have the potential to either detect particle acceleration in these shocks through its effect on the shock dynamics, or place a lower limit on the nonthermal pressure contributions in the undisturbed ICM. We briefly discuss implications for models of particle acceleration in collisionless shocks and the estimates of galaxy cluster masses derived from X-ray and Sunyaev-Zel'dovich effect observations.
\end{abstract}

Subject headings: cosmic rays — galaxies: clusters: general — galaxies: clusters: individual (A520, 1E 0657-06) intergalactic medium - shock waves - turbulence $-\mathrm{X}$-rays: galaxies: clusters

Online material: color figures

\section{INTRODUCTION}

Astrophysical collisionless shocks are the likely sources of the observed extrasolar high-energy cosmic rays (e.g., Bell 1978; Blandford \& Ostriker 1978). Within the solar system there are direct observations of cosmic ray acceleration in the Earth bow shock (e.g., Ellison et al. 1990) and in interplanetary shocks (e.g., Baring et al. 1997). Continuous observational effort has not yet yielded direct evidence for acceleration of cosmic ray nuclei in extrasolar collisionless shocks, although recently, tentative indirect evidence for such acceleration was identified in the morphology of the Tycho supernova remnant (Warren et al. 2005), and in the high-energy gamma-ray emission near the RX J1713.73946 remnant (Aharonian et al. 2007). The theory of diffusive shock acceleration (e.g., Blandford \& Eichler 1987 and references therein) predicts that the acceleration efficiency and the spectrum of accelerated particles depend on the Mach number and other parameters of the shock (e.g., Giacalone et al. 1997 and references therein). The presence of fossil cosmic rays in the preshock medium, e.g., from previous shocks, can also affect the acceleration efficiency (e.g., Kang et al. 2007; Kang \& Jones 2007 and references therein). We here argue that merging galaxy clusters are laboratories in which theories of cosmic ray acceleration in intermediate Mach number shocks can be tested. Observational constraints on particle acceleration in such shocks are especially interesting, as numerical simulations suggest that these are the source of a large fraction of the cosmic rays accelerated in galaxy clusters (e.g., Ryu et al. 2003).

\footnotetext{
1 Theoretical Astrophysics, Mail Code 130-33, California Institute of Technology, 1200 East California Boulevard, Pasadena, CA 91125.

2 Department of Astronomy, University of Texas, 1 University Station C1400, Austin, TX 78712.
}

Fossil cosmic rays, ${ }^{3}$ magnetic field, and turbulence may all contribute to the pressure of the intracluster medium (ICM), thereby modifying its hydrodynamic behavior. Such contributions may alter the interpretation of observations that ignore them. For example, X-ray observations have recently been used to estimate shock velocities in two merging clusters (see below), neglecting nonthermal components; improved estimates of shock velocities in these and other clusters may have to take into account the cosmic ray and other nonthermal contributions to the ICM pressure. Nonthermal pressure is also a source of systematic bias when cluster masses are estimated from X-ray and Sunyaev-Zel'dovich effect (SZE) measurements that are made assuming hydrostatic equilibrium between gravitational forces and thermal pressure gradients in the ICM (e.g., Enßlin et al. 1997; Rasia et al. 2006; Nagai et al. 2007a, 2007b and references therein). These nonthermal biases will limit the effectiveness of upcoming cluster surveys in the quest to place constraints on the expansion history of the universe.

Evidence for the nonthermal activity in clusters is growing. Observed radio and hard X-ray emission in clusters suggests the presence of relativistic electrons with Lorentz factors of $\sim 10^{4}$. This also suggests the presence of relativistic protons that could have been accelerated by the same mechanism that has accelerated the electrons. Direct evidence for cosmic-ray ions in the ICM is, however, still lacking. The nondetection in EGRET data of gamma-ray emission expected from neutral pion decay in cosmic ray collisions in the ICM (e.g., Reimer et al. 2003) has so far placed upper limits on the fraction of cosmic-ray pressure to

\footnotetext{
${ }^{3}$ Potential acceleration sites of fossil cosmic rays are past accretion and merger shocks, giant radio sources, supernovae, and turbulence in the ICM (e.g., Berezinsky et al. 1997).
} 
$\lesssim 20 \%-30 \%$ in several nearby rich clusters (Enßlin et al. 1997 ; Pfrommer \& Enßlin 2004). Most cluster atmospheres are also substantially magnetized, with typical field strengths of order a few $\mu \mathrm{G}$ out to $\mathrm{Mpc}$ radii (Carilli \& Taylor 2002; Govoni \& Feretti 2004; Govoni et al. 2006, and references therein). There is likely to be considerable variation in field strengths $(\sim 0.1-$ $40 \mu \mathrm{G})$ and topologies within clusters. Thus, while magnetic fields are likely to provide a significant contribution to the pressure in some regions (e.g., along some cold fronts; see Vikhlinin et al. 2001), the average energetics in the cluster outskirts is as yet unclear. Numerical simulations of cluster formation also suggest that subsonic gas motions (turbulence) contribute substantial nonthermal pressure in clusters (e.g., Norman \& Bryan 1999; Ricker \& Sarazin 2001; Nagai et al. 2003, 2007a, 2007b; Faltenbacher et al. 2005; Dolag et al. 2005; Rasia et al. 2006). Further investigations of nonthermal phenomena in clusters are hence critical for the success of upcoming X-ray and SZE cluster surveys, as our ability to estimate cluster masses hinges on a precise characterization of the nonthermal components.

In this work, we show that shock waves that form during merging of galaxy clusters can provide unique constraints on nonthermal processes in clusters. Recent Chandra X-ray observations have revealed that shock waves with Mach numbers $\mathcal{M} \approx 2-3$ that form during the merging of galaxy clusters are accompanied by distinct X-ray surface brightness and temperature discontinuities (see Markevitch \& Vikhlinin 2007 for a review). To date, a density and temperature jump along the symmetry axis of a cluster merger bow shock has been recovered in the cluster A520 (Markevitch et al. 2005) and in 1E 0657-56 (Markevitch et al. 2002; Markevitch 2006). The relative magnitudes of the density and temperature jump depend on the contribution of nonthermal components (e.g., cosmic rays, magnetic field, turbulence) to the pressure of the undisturbed, upstream ICM. They also depend on the efficiency of particle acceleration in the shock, on the escape of the accelerated particles from the shock, and on the amplification of turbulence in the shock.

Here we make the first attempt to use the X-ray observations of merging clusters to constrain their particle acceleration and the fractional contribution of nonthermal components to the pressure budget of the ICM. In $\S 2$, we model the hydrodynamic jump conditions and Mach number using the multifluid approximation. We model the effect of cosmic rays, a tangled magnetic field, and turbulence on observed gas jump conditions in the shock. In $\S 3$, we utilize this model to derive constraints on nonthermal components in merger shocks in galaxy clusters A520 and 1E 065756. We show that current observations can limit the efficiency of particle acceleration, and that the fractional contribution of nonthermal fluids to ICM pressure may be constrained with future, improved, X-ray spectroscopy and SZE observations. In $\S 4$, we discuss implications of these results in context of particle acceleration models and for the estimates of galaxy clusters masses with X-ray and SZE observations. In $\S 5$, we summarize our main conclusions.

\section{LIMITS ON PARTICLE ACCELERATION IN MERGER SHOCKS AND NONTHERMAL PRESSURE IN ICM}

\subsection{Shock Jump Conditions}

The ICM fluid consists of a thermal component (electrons and ions in mutual thermal equilibrium) and a number of nonthermal components (nonthermal cosmic rays and magnetic fields comoving with the thermal component). Turbulent gas motions and electromagnetic waves can also contribute pressure to the ICM.
We ignore the electromagnetic waves in the current treatment. We also temporarily ignore turbulence, but return to discuss its role on qualitative level in $\S 2.8$. The ICM fluid can be modeled with an adiabatic equation of state with an effective adiabatic index $\gamma .{ }^{4}$ In what follows, we use indices $u$ and $d$ to denote the shock upstream and downstream, respectively. Assuming cylindrical symmetry, conservation of density, momentum, and energy fluxes across the shock dictates jump conditions on the symmetry axis of the bow shock that read

$$
\begin{aligned}
\rho_{u} v_{u} & =\rho_{d} v_{d} \\
P_{u}+\rho_{u} v_{u}^{2} & =P_{d}+\rho_{d} v_{d}^{2} \\
v_{u}\left(\frac{1}{2} \rho_{u} v_{u}^{2}+\frac{\gamma_{u}}{\gamma_{u}-1} P_{u}\right) & =v_{d}\left(\frac{1}{2} \rho_{d} v_{d}^{2}+\frac{\gamma_{d}}{\gamma_{d}-1} P_{d}\right),
\end{aligned}
$$

where $\rho_{i}$ is the mass density of the thermal gas (the mass density of the nonthermal particles is negligible), $v_{i}$ is the fluid velocity, and $P_{i}$ is the total pressure $(i=\{u, d\})$.

The pressure is a sum of electronic, ionic, cosmic ray, and magnetic field contributions, $P_{i}=P_{e, i}+P_{i, i}+P_{\mathrm{CR}, i}+P_{B, i}$. The effective adiabatic index $\gamma_{i}$ equals

$$
\begin{aligned}
\frac{\gamma_{i}}{\gamma_{i}-1}= & \frac{1-\varepsilon_{\mathrm{nt}, i}}{2}\left(\frac{\gamma_{i, i}}{\gamma_{i, i}-1}+\frac{\gamma_{e, i}}{\gamma_{e, i}-1}\right) \\
& +\varepsilon_{\mathrm{CR}, i} \frac{\gamma_{\mathrm{CR}}}{\gamma_{\mathrm{CR}}-1}+\varepsilon_{B, i} \frac{\gamma_{B, i}}{\gamma_{B, i}-1}
\end{aligned}
$$

where $\varepsilon_{\mathrm{CR}, i} \equiv P_{\mathrm{CR}, i} / P_{i}$ and $\varepsilon_{B, i} \equiv P_{B, i} / P_{i}$ are, respectively, the fractional contribution of cosmic rays and magnetic fields to the total pressure, while $\varepsilon_{\mathrm{nt}, i}=\varepsilon_{\mathrm{CR}, i}+\varepsilon_{B, i}$ is the total fractional contribution of nonthermal pressure components. The temperature jump across the shock of the thermal electrons is then

$$
\tau \equiv \frac{T_{e, d}}{T_{e, u}}=\frac{\left(1-\varepsilon_{\mathrm{nt}, d}\right)\left[2 \gamma_{u} /\left(\gamma_{u}-1\right)-r^{-1}-1\right]}{\left(1-\varepsilon_{\mathrm{nt}, u}\right)\left[2 \gamma_{d} /\left(\gamma_{d}-1\right)-r-1\right]},
$$

where $r \equiv \rho_{d} / \rho_{u}$ is the compression ratio.

\subsection{Thermal Electron Relativistic Corrections}

Thermal ions are nonrelativistic in cluster shocks and thus $\gamma_{i, i}=5 / 3$. The adiabatic index of thermal electrons may differ from this value because of relativistic corrections. The adiabatic index of thermal electrons equals the ratio of the rest-frame pressure to internal energy density, $\gamma_{e}=1+P_{e} / \epsilon_{e}$, where $\epsilon_{e}$ is the internal electron energy density (e.g., Achterberg et al. 1984),

$\gamma_{e}=1+\frac{1}{3} \frac{\int_{0}^{\infty} 4 \pi p^{4}\left(p^{2} / c^{2}+m_{e}^{2}\right)^{-1 / 2} f_{e}(p) d p}{\int_{0}^{\infty} 4 \pi p^{2}\left[\left(p^{2} c^{2}+m_{e}^{2} c^{4}\right)^{1 / 2}-m_{e} c^{2}\right] f_{e}(p) d p}$,

where

$$
f_{e}(p) \propto \exp \left[-\frac{\left(p^{2} c^{2}+m_{e}^{2} c^{4}\right)^{1 / 2}}{k T_{e}}\right]
$$

is the thermal electron momentum distribution. Defining the electron temperature in units of the electron rest energy $\Theta_{e} \equiv$ $k T_{e} / m_{e} c^{2}$, the adiabatic index in equation (4) can conveniently

\footnotetext{
${ }^{4}$ We define the adiabatic indices via $\gamma=1+P / \epsilon$, where $\epsilon$ is the internal energy density. The adiabatic index thus calculated may differ from $\partial \ln P / \partial \ln \rho$.
} 
be expressed in terms of modified Bessel functions of the second kind (e.g., Kunik et al. 2003),

$$
\gamma_{e, i}=1+\Theta_{e, i}\left[3 \Theta_{e, i}+\frac{K_{1}\left(\Theta_{e, i}^{-1}\right)}{K_{2}\left(\Theta_{e, i}^{-1}\right)}-1\right]^{-1} .
$$

In the limit $\Theta_{e} \ll 1$, relevant to cluster merger shocks, the adiabatic index is $\gamma_{e}=5 / 3-(5 / 6) \Theta_{e}+\mathcal{O}\left(\Theta_{e}^{2}\right)$. Note that when $k T_{e, d}$ or $k T_{e, u}$ becomes comparable to $m_{e} c^{2}$, the right side of equation (3) depends on the electron temperatures. For example, if the nonthermal pressures are ignored (i.e., $\varepsilon_{\mathrm{nt}, i}=0$ ), a relativistic correction of about $10 \%$ applies to the expected temperature jump and inferred Mach number for conditions similar to those in the bow shock in $1 \mathrm{E} 0657-56\left(T_{u}=10 \mathrm{keV}\right.$ and $\left.r=3\right)$, while a correction of only about $1 \%$ applies for the lower temperature and weaker shock in $\mathrm{A} 520\left(T_{u}=5 \mathrm{keV}\right.$ and $\left.r=2.3\right)$.

\subsection{Fossil Cosmic Rays}

The undisturbed ICM may contain an intracluster population of fossil cosmic rays that could have been produced in the high Mach number accretion shocks $(\mathcal{M} \sim 10-100)$, in previous merger shocks, in active galactic nuclei, and/or in starburstassociated phenomena (see, e.g., Berezinsky et al. 1997; Fujita \& Sarazin 2001; Miniati et al. 2001; Gabici \& Blasi 2003; Sarazin 2004). After a merger bow shock passes a fluid element in the ICM, the fluid element will contain the original fossil cosmic rays, some of which may have been further accelerated in the shock. The shock may also accelerate new cosmic rays drawn from the thermal pool and accelerated in the shock for the first time.

The cosmic ray adiabatic index depends on whether their pressure is dominated by Newtonian or relativistic particles, which in turn depends on the details of the cosmic-ray spectrum (e.g., Achterberg et al. 1984). The effective cosmic-ray adiabatic index lies in the range $4 / 3 \leq \gamma_{\mathrm{CR}} \leq 5 / 3$; the electron cosmic rays are mildly or fully relativistic, while the protons that dominate the cosmic rays pressure can be Newtonian or relativistic. Thus, assuming that electron and proton cosmic rays are close to mutual equipartition, we expect $\gamma_{\mathrm{CR}}$ to range between $\gamma_{\mathrm{CR}} \approx 4 / 3$ (for relativistic electrons and protons) and $\gamma_{\mathrm{CR}} \approx 13 / 9$ (relativistic electrons and Newtonian protons; e.g., Konigl 1980). While in principle $\gamma_{\mathrm{CR}}$ may differ between the upstream and the downstream, when there is a significant population of upstream cosmic rays, it is reasonable to expect that these will also dominate the downstream, and thus that $\gamma_{\mathrm{CR}}$ is approximately constant across the shock.

In addition to being reaccelerated by the shock acceleration mechanism, fossil cosmic rays may be accelerated adiabatically during the shock compression of the magnetic field. However, shock compression may very well be nonadiabatic, as the Larmor radius of a mildly relativistic electron in a microgauss field, $r_{\mathrm{L}} \sim$ $10^{9} \mathrm{~cm}$, may be much larger than the transition layer over which the fluid density jumps. ${ }^{5}$ Therefore, it is possible that cosmic rays do not gain energy during compression. Nevertheless, the compression increases the cosmic ray number density and therefore its pressure by at least a factor of $r$. Thus, we express the fractional cosmic ray pressure in the downstream to be

$$
\varepsilon_{\mathrm{CR}, d}=r\left(\frac{P_{u}}{P_{d}}\right) \varepsilon_{\mathrm{CR}, u}+\varepsilon_{\mathrm{acc}}
$$

\footnotetext{
${ }^{5}$ If pressure across the transition layer is mediated not by cosmic rays but by plasma instabilities, the width of the transition layer will be of the order of the plasma skin depth of the thermal protons, which is $\delta \sim 10^{7} \mathrm{~cm}$. On the other hand, adiabatic acceleration will take place when $r_{\mathrm{L}} \ll \delta$.
}

where the first term is the pressure of the fossil cosmic rays if the energy of individual cosmic rays remains unchanged as they pass through the shock, and the second term is any additional pressure of cosmic rays that are accelerated in the shock. The acceleration term includes pressure due to accelerated particles from the upstream thermal pool that are added to the nonthermal pool, and also due to fossil nonthermal particles that are reaccelerated by a shock acceleration process and/or by adiabatic compression.

\subsection{The Cluster Magnetic Field}

The effect of the magnetic field on the shock depends on its strength and topology, both of which are constrained very poorly, especially in the cluster outskirts. Faraday rotation measurements suggest that the magnetic pressure can reach $\sim 10 \%$ of the thermal pressure in some clusters or regions within clusters, and that in others it is less than 1\% (e.g., Carilli \& Taylor 2002; Govoni \& Feretti 2004; Govoni et al. 2006). ${ }^{6}$ Given the large uncertainty in the field strength, we treat $\varepsilon_{B}$ as a free parameter in what follows.

We assume that the magnetic field of the undisturbed ICM is tangled and isotropic on scales relevant to the hydrodynamics of the cluster merger shock. This approximation allows us to use the unmagnetized form of the jump conditions, equation (1), to describe the effect of magnetic field on shock hydrodynamics. The approximate coherence length of the field in some galaxy clusters, $\sim 10 \mathrm{kpc}$, is shorter than the curvature scale of the merger shock, $\sim 100 \mathrm{kpc}$, so that one can average over the fluctuating field orientation near the shock. ${ }^{7}$ Furthermore, the measurements of density and temperature jumps across the shock are based on deprojection of the X-ray map assuming a cylindrical symmetry of a bow shock; thus, an averaging of observables on the shock curvature scale is implicit in the reported shock jump data.

Even if the upstream field is isotropic, the downstream field may be anisotropic as a result of a preferential amplification of the magnetic field component that is perpendicular to the shock normal, $B_{\perp}$. In this case the perpendicular component will still be isotropic in the plane of the shock, and can thus be parameterized by the ratio of the average perpendicular to parallel field energy densities $b \equiv\left\langle B_{\perp}^{2}\right\rangle / 2\left\langle B_{\|}^{2}\right\rangle$. The effective adiabatic index of the magnetic fluid, $\gamma_{B}$, which is defined as the parameter that correctly quantifies the behavior of the magnetic pressure in equation (2) and may depend on the orientation of the magnetic field, can be expressed in terms of the averages of the components $\mathcal{T}_{\mu \nu}^{\mathrm{em}}$ of the electromagnetic part of the energy-momentum tensor in the shock frame via

$$
\frac{\gamma_{B}}{\gamma_{B}-1}=\frac{\left\langle\mathcal{T}_{10}^{\mathrm{em}}\right\rangle}{\beta\left\langle\mathcal{T}_{11}^{\mathrm{em}}\right\rangle}
$$

Here, $\beta$ is the shock velocity in units of the speed of light, and $\Gamma \equiv\left(1-\beta^{2}\right)^{-1 / 2}$ is the Lorentz factor of the shock. Components of the energy-momentum tensor read

$$
\begin{aligned}
& \mathcal{T}_{11}^{\mathrm{em}}=\frac{1}{8 \pi}\left[\left(2 \Gamma^{2}-1\right) B_{\perp}^{2}-B_{\|}^{2}\right], \\
& \mathcal{T}_{10}^{\mathrm{em}}=\frac{1}{4 \pi} \Gamma^{2} \beta B_{\perp}^{2} .
\end{aligned}
$$

With these, for a nonrelativistic shock we obtain

$$
\gamma_{B}=\frac{4 b}{2 b+1} .
$$

\footnotetext{
${ }^{6}$ A magnetic field that is tangled on very small scales is not well constrained by the Faraday rotation measurements.

7 The same approximation was employed by Markevitch et al. (2005).
} 
The value of $\gamma_{B}$ in equation (10) equals $\gamma_{B}=2$ in a perpendicular shock $(b=\infty)$ and vanishes in a parallel shock $(b=0)$. If the field is isotropic on average in the shock upstream $(b=1)$, we recover the value $\gamma_{B, u}=4 / 3$. For an isotropic upstream field, the downstream field will have $b \geq 1$ and thus $4 / 3 \leq \gamma_{B, d} \leq 2$.

Relating $\varepsilon_{B, d}$ to $\varepsilon_{B, u}$ requires an understanding of the shock structure. But even if the shock structure is unknown, we can limit $\varepsilon_{B, d}$ in two extreme cases, when field generation in the shock itself (as may take place in unmagnetized shocks) is neglected. Assuming that magnetohydrodynamic jump conditions apply within the shock transition, and that the parallel and the perpendicular fields do not transform into each other, then $B_{\|, d}=$ $B_{\|, u}$ and $B_{\perp, d}=r B_{\perp, u}$, implying that $\varepsilon_{B, d}=\left(2 r^{2}-1\right)\left(P_{u} / P_{d}\right) \varepsilon_{B, u}$. The other extreme assumption, which was previously made by Markevitch et al. (2005), is that the field is isotropic and remains isotropic throughout the shock compression, in which case $\varepsilon_{B, d}=r^{4 / 3}\left(P_{u} / P_{d}\right) \varepsilon_{B, u}$, and thus the magnetic field behaves as a relativistic or photon gas that is adiabatically compressed in the shock. We expect $\varepsilon_{B, d}$ to lie between these two limits, assuming that no new field is generated within the shock.

\subsection{Escape of the Highest Energy Cosmic Rays}

Another process that may affect the shock and that we have ignored so far is the escape of the highest energy cosmic rays that are accelerated in the shock. This process may take place if the shock efficiently produces a hard cosmic-ray spectrum, and the highest energy cosmic rays can escape and remove energy from the shock transition (see, e.g., Achterberg et al. 1984). This energy leakage can be parameterized by the fraction $Q$ of the incoming energy flux that escapes the shock. The leakage affects the shock jump conservation relations such that the left-hand side of the energy flux conservation relation - the last of equation (1) must be multiplied by the factor $1-Q$. With this, equation (3) generalizes into

$\tau=\frac{\left(1-\varepsilon_{\mathrm{nt}, d}\right)\left[2(1-Q) \gamma_{u} /\left(\gamma_{u}-1\right)-r^{-1}-1+Q r /(r-1)\right]}{\left(1-\varepsilon_{\mathrm{nt}, u}\right)\left[2 \gamma_{d} /\left(\gamma_{d}-1\right)-r-1+Q r^{2} /(r-1)\right]}$.

The effect of escape is similar to the effect of particle acceleration, as it removes a fraction of the incoming energy from the downstream thermal fluid, thereby reducing the temperature jump. Note, however, that if the measured value of $\tau$ is below the one expected in a thermal shock, the deviation from the thermal temperature jump can only partially be attributed to the escape, since when $\varepsilon_{\text {acc }}=0$, the value of $Q$ must by definition be zero.

\subsection{Sensitivity of the Temperature Jump}

We have one relation (eq. [3] or its generalized form, eq. [11]) and several unknown parameters describing the upstream and downstream contribution of the cosmic rays and magnetic field to the fluid pressure. Without making assumptions about the nature of the nonthermal fluids, given a measurement of the density jump $r$ and the temperature jump $\tau$, we can place constraints in the joint parameter space spanned by these parameters, but cannot recover the fractional pressures themselves. In addition, when only soft X-ray data are available (as from Chandra and $X M M$-Newton), the uncertainties in $\tau$ typically exceed the uncertainties in $r$. Therefore, before we proceed to explore the joint parameter space, we discuss the effect of the variation of various parameters on $\tau$, assuming that $r$ is accurately measured and is held fixed. The temperature jump is most sensitive to the fractional pressure in cosmic rays accelerated or reaccelerated in the shock, $\varepsilon_{\text {acc }}$. A nonzero value of $\varepsilon_{\text {acc }}$ reduces the value of $\tau$, e.g., in the ideal case in which the upstream nonthermal pressure vanishes, $\varepsilon_{\mathrm{nt}, u}=0$, we find that $\tau$ drops by about a factor of 2 between $\varepsilon_{\text {acc }}=0$ and $\varepsilon_{\text {acc }}=0.3(r=2-3)$. The reason for this sensitive dependence is that the production of accelerated particles in the shock saps a fraction of the incoming energy flux out of the thermal component of the downstream, thereby reducing $\tau$. Increasing $Q$ results in a similar effect, for similar reason, on $\tau$.

The effect on the temperature jump of changing $\varepsilon_{\mathrm{CR}, u}$ and $\varepsilon_{B, u}$ is more subtle, since a high nonthermal pressure in the shock upstream implies that there will also be a high nonthermal pressure in the downstream. The value of $\tau$ is determined mostly by the balance between the upstream and downstream fractional component of the thermal pressure (both decrease with upstream nonthermal pressure) and by the balance of the effective upstream and downstream adiabatic index. The interplay between these effects mostly cancels, and therefore $\tau$ depends only weakly on the upstream nonthermal pressure. Increasing $\varepsilon_{\mathrm{CR}, u}$ while keeping the rest of the parameters constant typically increases $\tau$ slowly for $r=2-3$. For example, assuming that the magnetic pressure vanishes $\left(\varepsilon_{B, u}=0\right)$ and that $\gamma_{\mathrm{CR}}=4 / 3, \tau$ increases by a factor of $\approx 1.2$ between $\varepsilon_{\mathrm{CR}, u}=0$ and $\varepsilon_{\mathrm{CR}, u}=0.3$ for $r=2.3$ and $\varepsilon_{\mathrm{acc}}=0$. Having a constant $\varepsilon_{\text {acc }}=0.15$ reduces the change in $\tau$ to a factor of $\approx 1$.1. Increasing $\varepsilon_{B, u}$ also mostly results in a slightly larger temperature jump. If the perpendicular field is strongly amplified in the shock, $\gamma_{B}$ jumps in the shock, thereby increasing the effective downstream adiabatic index $\gamma_{d}$, and thus increasing $\tau$. If on the other hand we treat the field as a relativistic gas, then $\varepsilon_{B, u}$ and $\tau$ are positively correlated for $\varepsilon_{\text {acc }}=0$ and are weakly anticorrelated for $\varepsilon_{\text {acc }} \approx 0.15$.

In conclusion, $\tau$ is strongly anticorrelated with $\varepsilon_{\text {acc }}$ and is typically weakly positively correlated with $\varepsilon_{\mathrm{CR}, u}$ and $\varepsilon_{B, u}$. Therefore, an accurate measurement of $\tau$ and $r$ can tightly constrain $\varepsilon_{\text {acc }}$. If the measured value of $\tau$ falls below that expected in a purely thermal hydrodynamic shock given a precise measurement of $r$, then a nontrivial lower and upper bound can be placed on $\varepsilon_{\text {acc }}$, but such a constraint cannot be obtained for $\varepsilon_{\mathrm{CR}, u}$ and $\varepsilon_{B, u}$. If, on the other hand, the measured value of $\tau$ is higher than that expected in a purely thermal hydrodynamic shock, only an upper limit on $\varepsilon_{\text {acc }}$ can be placed, i.e., $\varepsilon_{\text {acc }}=0$ remains viable. Then, however, the measurement places a lower limit on the upstream nonthermal pressure. However, based on the measurement of $r$ and $\tau$ alone, one cannot separate the partial contributions of the cosmic-ray and magnetic components.

\subsection{Implications for Shock Velocity Estimation}

The velocity of cluster merger shock is of great interest, since it is a stepping stone toward relating the dynamics of the ICM to the dynamics of the dark matter in galaxy cluster mergers (e.g., Hayashi \& White 2006; Farrar \& Rosen 2007; Milosavljević et al. 2007; Springel \& Farrar 2007). Typically, the shock Mach number is inferred from the compression ratio $r$, which can be measured relatively accurately in X-ray maps, under the strict assumption that all pressures are thermal (the observed constraints on $\tau$ are typically used for a consistency check with this assumption). Taking the nonthermal pressure into account (but ignoring cosmic-ray escape; see $\S 2.5$ ), the Mach number is given by

$$
\mathcal{M}^{2}=\frac{2 r \gamma_{u} /\left(\gamma_{u}-1\right)-2 \gamma_{d} /\left(\gamma_{d}-1\right)}{\gamma_{g, u}\left(1-\varepsilon_{\mathrm{nt}, u}\right)\left(1-r^{-1}\right)\left[2 \gamma_{d} /\left(\gamma_{d}-1\right)-r-1\right]},
$$

where $\gamma_{g, u}=5 / 3$ is the adiabatic index of the upstream thermal gas, for which relativistic corrections are negligible. This relation implies that high nonthermal pressure in the upstream (downstream) 
increases (decreases) $\mathcal{M}$. For example, assuming that $\varepsilon_{\mathrm{nt}, u}=0$ and cosmic rays are rather efficiently accelerated in the shock, $\varepsilon_{\text {acc }}=0.15$, the inferred value of the Mach number must be revised by $\approx 10 \%-20 \%$ downward of the value inferred for a thermal shock, when $r$ is in the range 2-3.

If, in addition to $r$, the value of $\tau$ is accurately measured, the constraints that can be placed on the Mach number are tighter, since the upstream and downstream nonthermal components are no longer entirely free. In this case, when $r$ and $\tau$ are held constant, a larger nonthermal pressure results in a higher Mach number. For example, if the nonthermal component behaves as relativistic gas $\left(\gamma_{\mathrm{nt}}=4 / 3\right)$ and $r$ and $\tau$ are related as they would be in a purely thermal shock, $\tau=(4 r-1) / r(4-r)$, the shock Mach number equals

$$
\mathcal{M}^{2}=\frac{3(4 r-1)\left(1-\varepsilon_{\mathrm{nt}, u}\right) /(4-r)+5 r+3 r \varepsilon_{\mathrm{nt}, u}-8}{\gamma_{u}\left(1-\varepsilon_{\mathrm{nt}, u}\right)\left(1-r^{-1}\right)(7-r)},
$$

in which case $\mathcal{M}$ increases by $\approx 10 \%$ for $\varepsilon_{\mathrm{nt}, u}=0.3$, compared to the purely thermal shock, for $r$ in the range 2-3.

\subsection{Turbulence}

In addition to the cosmic rays and the magnetic field, turbulence also contributes pressure to the ICM. Turbulence in the ICM is expected to be driven by gravitational clustering (accretion and merging), and by outflows associated with active galactic nuclei. Hydrodynamical simulations of galaxy cluster formation in which the ICM is treated as an ideal fluid universally demonstrate that turbulent pressure in the ICM is nonnegligible, and that its contribution to the total pressure is an increasing function of radius from the center of the cluster. The fractional turbulent pressure measured in the simulations is $\varepsilon_{\text {tur }} \sim 0.06-0.36$ (Norman $\&$ Bryan 1999), $\varepsilon_{\text {tur }} \sim 0.05-0.1$ (Ricker \& Sarazin 2001), $\varepsilon_{\text {tur }} \sim$ 0.04-0.09 (Nagai et al. 2003; Faltenbacher et al. 2005), and $\varepsilon_{\text {tur }} \sim 0.05-0.3$ (Dolag et al. 2005). ${ }^{8}$ Spatially resolved gas pressure maps obtained from XMM-Newton observations of the Coma galaxy cluster show a scale-invariant pressure fluctuation spectrum on the scales of 40 to $90 \mathrm{kpc}$, which was analyzed to place a lower limit on the fractional turbulent pressure of $\varepsilon_{\text {tur }} \gtrsim 0.1$ (Schuecker et al. 2004). ${ }^{9}$

The physics of the interaction of a shock wave with a turbulent upstream is complex and poorly understood even in unmagnetized, ideal fluids. The primary theoretical uncertainties are the amount of amplification of turbulence in the shock and the affect of turbulence on the shock structure. The shock transition becomes nonplanar in the presence of turbulence (e.g., Rotman 1991), and this nonplanarity affects the local shock jump conditions that short-wavelength fluctuations experience while crossing the shock (e.g., Zank et al. 2002). Different analytical approximations (e.g., the rapid distortion theory and the local interaction analysis) and direct numerical simulations do not always agree with each other (e.g., Andreopoulos et al. 2000 and references therein). Therefore, we do not attempt to include turbulent pressure in our quantitative calculations. However, since turbulent pressure can affect the observed jump conditions, we discuss it qualitatively using a simple model (Lele 1992) based on the rapid distortion theory applied to homogeneous turbulence (see, e.g., Batchelor 1953; Jacquin et al. 1993).

\footnotetext{
8 Hydrodynamic simulations of intracluster turbulence were also recently carried out by Fujita et al. (2004), Subramanian et al. (2006), and Vazza et al. (2006).

9 Turbulence in the ICM can be detected in other clusters given an X-ray detector with high spectral resolution ( Inogamov \& Sunyaev 2003; Sunyaev et al. 2003).
}

Lele (1992) derives the averaged density, momentum, and energy conservation equations of ideal fluid in the shock frame (his eqs. [9]-[11]). Assuming homogeneous turbulence and a cylindrically symmetric distribution of turbulent fluctuations, the conservation equations are reduced to the form of equation (1) with a turbulent pressure and effective adiabatic index of

$$
P_{\mathrm{tur}, i}=\overline{\rho_{i}} \widetilde{v}_{\|, i}^{\prime \prime} v_{\|, i}^{\prime \prime}, \quad \gamma_{\mathrm{tur}}=\frac{3+2 b_{i}}{1+2 b_{i}}
$$

Here, the notation is such that any fluctuating quantity $f$ is decomposed in two ways: $f=\bar{f}+f^{\prime}=\tilde{f}+f^{\prime \prime}$, with $\bar{f}$ denoting the average value of the quantity, $\tilde{f} \equiv \overline{\rho f} / \bar{\rho}$ denoting the mass-weighted average, and $f^{\prime}$ and $f^{\prime \prime}$ denoting the corresponding fluctuating parts, while as before, $i=(u, d)$. Just as we did for the magnetic field (see $\S 2.4$ ), we use cylindrical symmetry to parameterize the turbulent field with a single parameter $b_{i}=v_{\perp, i}^{\prime \prime} v_{\perp, i}^{\prime \prime} /\left(2 v_{\|, i}^{\prime \prime} v_{\|, i}^{\prime \prime}\right)$, which in the isotropic case equals unity.

The temperature jump $\tau$ and the shock Mach number $\mathcal{M}$ can be calculated for a given upstream turbulent pressure fraction $\varepsilon_{\text {tur }, u} \equiv P_{\text {tur }, u} / P_{u}$ and anisotropy parameter $b_{u}$ if the amplification of the turbulence in the shock is known. ${ }^{10}$ Lele (1992) uses the rapid distortion theory to derive the shock amplification. This approximation assumes that the mean turbulent flux amplitudes are much smaller than their mean flow counterparts, that turbulent fluctuations cross the shock much faster than the corresponding eddy turnaround times, and that the mean flow does not vary much on the length scale of an eddy. In particular, the otherwise planar shock transition is assumed not to have been distorted, and rendered nonplanar, by the fluctuations. In this theory, the parallel and perpendicular turbulence is amplified according to (Lele 1992)

$$
\begin{aligned}
\frac{v_{\|}^{\prime \prime} v_{\| u}^{\prime \prime}}{\widetilde{v_{\|}^{\prime \prime} v_{\| d}^{\prime \prime}}} & =\frac{3}{4} r^{2}\left[\frac{1}{r^{2}-1}+\frac{r^{2}-2}{\left(r^{2}-1\right)^{3 / 2}} \tan ^{-1} \sqrt{r^{2}-1}\right] \\
& \approx \frac{6 r-1}{5} \\
\widetilde{v_{\perp}^{\prime \prime} v_{\perp}^{\prime \prime}} & =\frac{3}{8}\left[1-\frac{1}{r^{2}-1}+\frac{r^{4}}{\left(r^{2}-1\right)^{3 / 2}} \tan ^{-1} \sqrt{r^{2}-1}\right] \\
& \approx \frac{r+1}{2},
\end{aligned}
$$

which in the case of isotropic upstream turbulence, $b_{u}=1$ and $\gamma_{\text {tur }, u}=5 / 3$, yields effective adiabatic index and downstream pressure fraction for turbulence

$$
\begin{aligned}
\gamma_{\mathrm{tur}, d} & \approx \frac{23 r+2}{11 r+4}, \\
\varepsilon_{\mathrm{tur}, d} & \approx \frac{\varepsilon_{\mathrm{tur}, u}(6 r-1)}{5 \tau\left(1-\varepsilon_{\mathrm{tur}, u}\right)+\varepsilon_{\mathrm{tur}, u}(6 r-1)} .
\end{aligned}
$$

In this simple model of amplification of turbulence in the shock, the effect of a preexisting turbulent component is similar to that of the preexisting cosmic rays and magnetic pressure, namely, the upstream turbulence is only weakly, positively correlated with $\tau$ and $\mathcal{M}$. For example, assuming that turbulence is the only nonthermal component, the temperature jump increases by a factor of $\approx 1.15$, and $\mathcal{M}$ increases by a factor of $\approx 1.3$ between $\varepsilon_{\text {tur }, u}=0$ and $\varepsilon_{\text {tur }, u}=0.3$ for compression ratio $r=2.3$. Very similar results are obtained if instead of the rapid distortion theory, we employ

\footnotetext{
${ }^{10}$ In the case of isotropic turbulence $\varepsilon_{\text {tur }}=\gamma_{g} \mathcal{M}_{\text {tur }}^{2} /\left(3+\gamma_{g} \mathcal{M}_{\text {tur }}^{2}\right)$, where $\mathcal{M}_{\text {tur }}$ is the turbulence Mach number and $\gamma_{g}$ is the thermal gas adiabatic index.
} 
the linear interaction analysis to calculate the jump conditions (e.g., Lee et al. 1993, 1997). Therefore, we conclude that for a weak, subsonic, unmagnetized turbulence of the ICM, the effect of turbulence on the thermal gas temperature jump of the shock is similar to that expected in the presence of other nonthermal components.

\section{RESULTS}

\section{1. $A 520$}

Markevitch et al. (2005) analyze a 67 ks observation with Chandra ACIS-I of the bow shock in the galaxy cluster merger A520 at $z=0.203$ and estimate the density and temperature jump along the axis of symmetry of the shock. The upstream and downstream temperatures are, respectively, $T_{u}=4.8_{-0.8}^{+1.2} \mathrm{keV}$ and $T_{d}=$ $11.5_{-3.1}^{+6.7} \mathrm{keV}$, while the density jump is $r=2.3 \pm 0.3$ (all errors are at the $90 \%$ confidence levels). These values are consistent with a shock Mach number of $\mathcal{M} \approx 2$. We use equation (3) to explore the constraints that can be placed on the acceleration of particles in the shock and on the fractional pressure in the nonthermal components in the shock upstream, and equation (11) to constrain energy leakage from the shock in A520.

The predicted downstream temperature in the case of a pure thermal gas, $T_{d}=10 \mathrm{keV}$ for $T_{u}=4.8 \mathrm{keV}$ and $r=2.3$, is consistent with the measured value. However, given that the expected temperature is below the median measured temperature, little room is left for significant particle acceleration in the shock. To place constraints on $\varepsilon_{\text {acc }}$, we first take $\varepsilon_{B, i}=Q=0$, and carry out a Monte Carlo search in the remaining parameter space $\left(\varepsilon_{\mathrm{CR}, u}, \varepsilon_{\mathrm{acc}}\right)$. We draw a large set $\left(10^{5}\right)$ of the observed parameters (density and temperature jump) from the observed distributions. ${ }^{11}$ For each set of observed values, we find all the combinations of $\left(\varepsilon_{\mathrm{CR}, u}, \varepsilon_{\mathrm{acc}}\right)$ in the domain $\left(0<\varepsilon_{\mathrm{CR}, u}<0.3,0<\varepsilon_{\mathrm{acc}}<0.25\right)$ that are compatible with the observations. For each point in the $\left(\varepsilon_{\mathrm{CR}, u}, \varepsilon_{\mathrm{acc}}\right)$ plane, we calculate the number of instances that the corresponding shock is compatible with the generated "observed" values of $r$ and $\tau$. The resulting number, properly normalized, provides the Bayesian likelihood of the shock being characterized by a given pair $\left(\varepsilon_{\mathrm{CR}, u}, \varepsilon_{\mathrm{acc}}\right)$, assuming a uniform prior in the domain considered here.

Figure 1 shows the probability distribution for $\left(\varepsilon_{\mathrm{CR}, u}, \varepsilon_{\mathrm{acc}}\right)$ for the relativistic cosmic rays $\left(\gamma_{\mathrm{CR}}=4 / 3\right)$. The two contours divide the plane so that the cumulative probability constrained above each is 0.33 and 0.05 of the total. The figure shows that $\varepsilon_{\text {acc }} \lesssim 0.1$ at $95 \%$ confidence levels for any value of $\varepsilon_{\mathrm{CR}, u}$, and that current observations do not provide a constraint on $\varepsilon_{\mathrm{CR}, u}$. Carrying out a similar analysis for relativistic electrons and Newtonian proton cosmic rays $\left(\gamma_{\mathrm{CR}}=13 / 9\right)$, which would be expected if the cosmic-ray pressure were dominated by particles with typical energies of $10^{1}-10^{4}$ times the thermal energy, yields an upper limit of $\varepsilon_{\text {acc }} \lesssim 0.15$. Finally, the limit on the efficiency of the acceleration of Newtonian cosmic rays, $\gamma_{\mathrm{CR}}=5 / 3$, as expected if the cosmic rays are accelerated only to several times the thermal temperature, is $\varepsilon_{\text {acc }} \lesssim 0.25$. Naturally, allowing for $Q>0$ when deriving the constraints on $\varepsilon_{\text {acc }}$ yields a tighter upper limit on $\varepsilon_{\text {acc }}$. The minor effect of varying upstream magnetic field and upstream cosmic ray pressure on the limits that can be placed on $\varepsilon_{\text {acc }}$ is explored in Figure 2.

Repeating the same analysis for various values of $0<\varepsilon_{B, u}<0.3$ while assuming $\varepsilon_{\mathrm{CR}, u}=0$, or taking a constant $\varepsilon_{\mathrm{CR}, u}$ and a flat

\footnotetext{
11 We approximate each observed distribution by two half-Gaussians that peak at the median observed value and satisfy the $90 \%$ confidence range reported by Markevitch et al. (2005).
}



FIG. 1.-Probability distribution of $\left(\varepsilon_{\mathrm{CR}, u}, \varepsilon_{\mathrm{acc}}\right)$ for relativistic cosmic rays in the bow shock in the galaxy cluster A520, assuming $\varepsilon_{B, u}=0$ and $\varepsilon_{\mathrm{CR}, u}<0.3$. The contours divide the plane so that the cumulative distribution above the contour includes only 0.33 (lower contour) and 0.05 (upper contour) of the total probability. The probability is calculated via a Monte Carlo simulation (see text). [See the electronic edition of the Journal for a color version of this figure.]

prior on $\varepsilon_{B, u}$ in the same range, for the two limiting forms magnetic field behavior at the shock $\left(B_{\perp, d}=r B_{\perp, u}\right.$ and $B_{d}=r^{2 / 3} B_{u}$; see $\S 2.4$ ), yields a similar upper limit on $\varepsilon_{\text {acc }}$. The reason for the weak impact of magnetic pressure on the constraints that can be placed on particle acceleration is the strong dependence of $\tau$ on $\varepsilon_{\text {acc }}$ and its weak dependence on $\varepsilon_{B, u}$. Therefore, just as we found for $\varepsilon_{\mathrm{CR}, u}$, current observations do not provide a significant constraint on $\varepsilon_{B, u}$.

We also carried out the same analysis assuming no particle acceleration, $\varepsilon_{\mathrm{acc}}=0$, but allowing for an escape of cosmic rays from the shock, in order to constrain energy leakage from the shock, $Q$. Although this scenario is artificial (since for $\varepsilon_{\text {acc }}=0$ we also expect $Q=0$ ), this analysis provides an upper limit to the value of $Q$. We find that the observation of A520 limit $Q \lesssim 0.1$ in its merger shock.

The dependence of the temperature jump in equation (3) on $\varepsilon_{\mathrm{CR}, u}$ and $\varepsilon_{B, u}$ is weak. Therefore, current observations do not put a significant constraint the presence of a relativistic component in the premerger ICM. Figure 1 shows that the purely thermal scenario is not the most likely one according to current observations, although it is consistent within $1 \sigma$. Future observations with tighter constraints on $\tau$ (e.g., improved X-ray spectroscopy across the bow shock) will reduce the volume of the allowed phase space and therefore may exclude the purely thermal scenario, $\varepsilon_{\text {acc }}=$ $\varepsilon_{\mathrm{CR}, u}=0$. If the measured value of $\tau$ falls below the thermal prediction, our analysis will yield a lower limit on $\varepsilon_{\text {acc }}$ to accompany the current upper limit. If the measured value of $\tau$ falls above the thermal prediction, the measurement will imply a lower limit on $\varepsilon_{\mathrm{CR}, u}$. For example, if we artificially reduce the present measurement uncertainties in $T_{u}, T_{d}$, and $r$ by a factor of 3 while assuming that the mean values of these observables remained unchanged, the data would require $\varepsilon_{\text {acc }}<0.05$ and $\varepsilon_{\text {nt. } u}>0.05$ at $95 \%$ confidence levels. However, decoupling $\varepsilon_{\mathrm{nt}, u}$ into its cosmic ray and magnetic field components cannot be accomplished given a measurement of $r$ and $\tau$ alone.

From the density jump data alone and assuming a purely thermal shock, Markevitch et al. (2005) estimate the Mach number of the merger shock in A520 to be $\mathcal{M}=2.1_{-0.3}^{+0.4}$. As we discuss in $\S 2.7$, the likely presence of a nonnegligible nonthermal pressure requires a modification of the Mach number estimate as in 


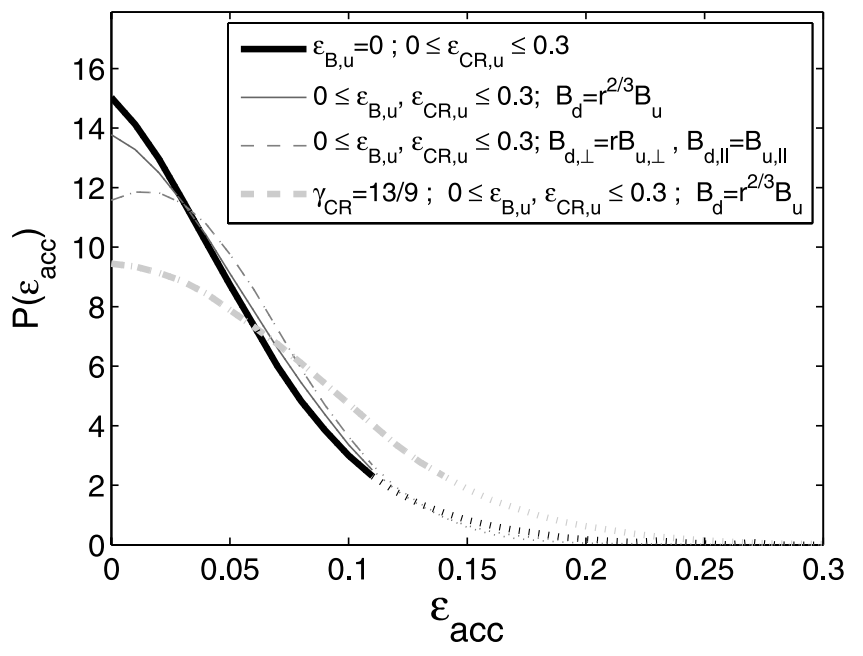

FIG. 2.-Marginalized probability distribution of $\varepsilon_{\text {acc }}$ for several different nonthermal contributions to the gas pressure. The probability is calculated via a Monte Carlo simulation (see text) for various ranges of flat priors on $\varepsilon_{\mathrm{CR}, u}$ and $\varepsilon_{B, u}$, and various levels of magnetic field amplification in the shocks, as indicated in the legend. The cosmic rays are relativistic $\left(\gamma_{C R}=4 / 3\right)$, unless noted otherwise. The solid and dashed lines contain $90 \%$ of the distributions (the rest is in the dotted-line tail). This figure shows that the limits on $\varepsilon_{\text {acc }}$ are insensitive to the assumptions and the priors that we choose for the upstream nonthermal components. [See the electronic edition of the Journal for a color version of this figure.]

equation (12); adding a nonthermal pressure in the upstream increase the Mach number. Figure 3 shows the Mach number probability for several scenarios in which a nonthermal fluid is present, with a fractional contribution to the pressure that is allowed by the present observations. The true value of the Mach number depends on the fractional nonthermal pressure and the amplification of the magnetic field and turbulence in the shock. For example, for $\varepsilon_{\mathrm{CR}, u}=\varepsilon_{B, u}=0.15$ and assuming significant magnetic field amplification in the shock, the true Mach number can be as high as $\mathcal{M} \approx 2.7$.

\section{2. $1 E 0657-56$}

Gas properties across the merger shock in 1E 0657-56 (the "bullet" cluster at $z=0.296$ ) were measured by Markevitch (2006) using a $500 \mathrm{ks}$ observation with Chandra ACIS-I. They find a density jump of $r \approx 3$, which corresponds to a Mach number of $\mathcal{M} \approx 3$. The measured temperatures are $T_{u} \approx 9 \mathrm{keV}$ and a lower limit $T_{d}>32 \mathrm{keV}$ at $1 \sigma$ confidence levels. The shock in 1E 0657-56 is stronger than that in A520 and is thus more propitious for detecting particle acceleration. Unfortunately, the high downstream temperature $T_{d}$ complicates an accurate measurement of the temperature jump with the high-resolution X-ray telescopes Chandra and XMM-Newton.

Markevitch (2006) does not report the errors on some of the measurements, and therefore we cannot quantitatively constrain the presence of nonthermal components in the shock upstream and downstream. Figure 4 shows the model prediction for $T_{d}$ as a function of $\varepsilon_{\mathrm{CR}, u}$ and $\varepsilon_{\mathrm{acc}}$ for the case of relativistic cosmic rays, $\gamma_{\mathrm{CR}}=4 / 3$, assuming that $\varepsilon_{B, u}=Q=0$ and taking $r=3$ and $T_{u}=9 \mathrm{keV}$. The figure shows that constraints that can be made in 1E $0657-56$ are qualitatively similar to, although less stringent than, those in A520. The minimum value of the temperature jump $\tau$ allowed by the measurement is high and barely consistent with a purely thermal shock. It does not leave much room for particle acceleration. We tentatively infer $\varepsilon_{\text {acc }}<0.15$, since larger $\varepsilon_{\text {acc }}$ implies $T_{d} \lesssim 20 \mathrm{keV}$. A similar low-temperature jump

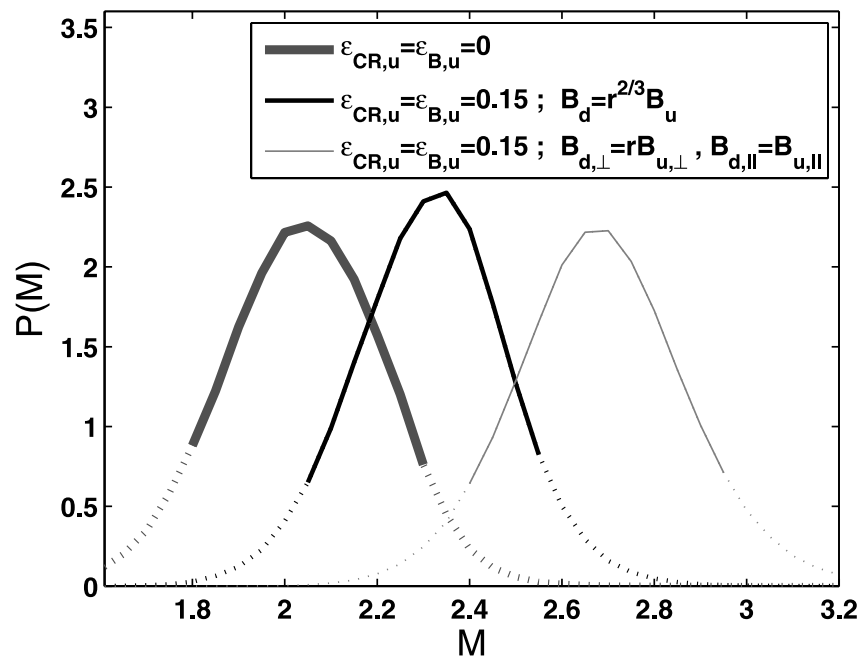

FIG. 3.-Mach number probability distribution based on the observed density and temperature jumps across the shock in A520 for several different contributions of nonthermal components to the gas pressure. The solid lines contain $90 \%$ of the distributions (the rest is in dotted-line tails). In all cases the cosmic rays are relativistic and the upstream magnetic field is isotropic $\left(\gamma_{C R}=\gamma_{B, u}=4 / 3\right)$. The legend indicates the fractional upstream nonthermal pressure and the shock amplification of the field in each case. [See the electronic edition of the Journal for a color version of this figure.]

is explored by Markevitch (2006) in the context of adiabatic electron heating (i.e., only the protons are shocked) and is rejected with a confidence of $95 \%$.

The nonthermal components may affect also the Mach number of the bullet cluster merger shock, which recently stirred a discussion about its compatibility with standard cosmological models (Hayashi \& White 2006; Farrar \& Rosen 2007; Milosavljević et al. 2007; Springel \& Farrar 2007). Markevitch (2006) finds $\mathcal{M}=3.0 \pm 0.4$, which corresponds to $r=3.0_{-0.23}^{+0.17}$, assuming no nonthermal contribution and neglecting relativistic corrections due to the high electron temperature. Allowing for cosmic rays with pressure up to equipartition, $0<\varepsilon_{\mathrm{CR}, u}<0.3$ and $\varepsilon_{B}=0$, while requiring temperature and density jump consistent with observations, $T_{d}>20 \mathrm{keV}$ and $2.77<r<3.17$, yields the limits

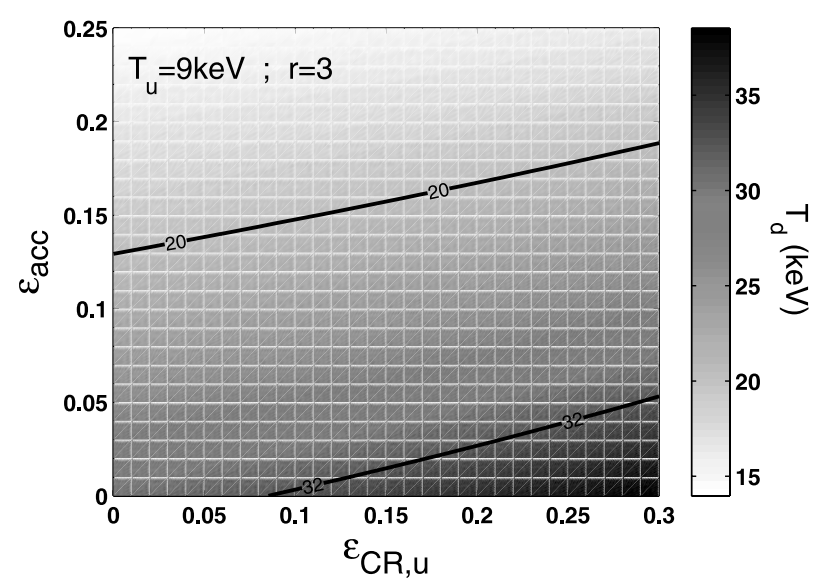

FIG. 4. $-T_{d}$ as a function of $\varepsilon_{\mathrm{CR}, u}$ and $\varepsilon_{\mathrm{acc}}\left(\gamma_{\mathrm{CR}}=4 / 3\right)$ for $r=3$ and $T_{u}=$ $9 \mathrm{keV}$, which correspond to the values reported for the bullet cluster 1E $0657-$ 56 . The 32 and $20 \mathrm{keV}$ contours are the $1 \sigma$ and $2 \sigma$ lower limits on $T_{d}$ as measured by Markevitch (2006) (the $2 \sigma$ limit is a rough estimate derived from the plots in Markevitch 2006). [See the electronic edition of the Journal for a color version of this figure.] 
$2.3<\mathcal{M}<3.7$. The lower bound is obtained for $r=2.77, \varepsilon_{\mathrm{acc}}=$ $0.07, \varepsilon_{\mathrm{CR} . u}=0$, and $T_{d}=20 \mathrm{keV}$. The upper bound corresponds to $r=3.17, \varepsilon_{\mathrm{acc}}=0, \varepsilon_{\mathrm{CR}, u}=0.3$, and $T_{d}=45 \mathrm{keV}$.

\section{DISCUSSION}

\subsection{Particle Acceleration in Collisionless Shocks}

Current measurements place constraints on the efficiency of particle acceleration in cluster merger shocks. This offers a unique opportunity to test models of particle acceleration in astrophysical collisionless shocks. Estimating the acceleration efficiency in collisionless shocks is a difficult problem, which is severely complicated by the fact that it remains unknown which among a number of possibilities is the primary acceleration mechanism. Even in the leading candidate mechanism, the diffusive shock acceleration, estimates of acceleration efficiency range widely because of a number of fundamental theoretical uncertainties, concerning the fraction of thermal particles that are injected into the acceleration process (e.g., Malkov \& Drury 2001 and references therein), the magnetic field geometry (i.e., parallel vs. perpendicular), the nonlinear influence of the accelerated particles on the hydrodynamic profile of the shock wave (e.g., Drury \& Völk 1981; Achterberg et al. 1984; Giacalone et al. 1997; Kang et al. 2002, 2007), and the amplification of the magnetic field by plasma instabilities (e.g., Lucek \& Bell 2000; Bell \& Lucek 2001; Schlickeiser \& Shukla 2003; Bell 2004, 2005; Schekochihin et al. 2005; Medvedev et al. 2006). Therefore, any estimate of particle acceleration efficiency is strongly affected by the specific assumptions and approximations employed in a self-consistent shock model. Here we do not attempt to carry out a detailed comparison of our results with various scenarios for particle acceleration. But, to demonstrate the power of the constraints that can be obtained from merger shock dynamics, we discuss our results in the context of the predictions of the particle acceleration model of Kang et al. (2007; see also Kang \& Jones 2007).

A variety of investigations point to a strong dependence of acceleration efficiency on the shock Mach number, whereby stronger shocks produce higher efficiencies. Kang et al. (2007) report an investigation of acceleration efficiency behavior in diffusive shock acceleration simulations in quasi-parallel shocks with a Bohm diffusion coefficient, a self-consistent treatments of particle injection from the thermal pool into the acceleration process, and Alfvén wave propagation. In their Figure 5, Kang et al. (2007) plot the dependence of the acceleration efficiency $\eta(\mathcal{M})$ on the shock Mach number $\mathcal{M}$, where $\eta$ is calculated as the ratio of the integrated cosmic-ray energy to the bulk kinetic energy that has passed through the shock. ${ }^{12}$ In the limit $\varepsilon_{\text {acc }} \ll 1$, the parameter $\eta$ in Kang et al. is related to our $\varepsilon_{\text {acc }}$ via

$$
\eta \approx \frac{3}{10} \frac{\left(\mathcal{M}^{2}+3\right)\left(5 \mathcal{M}^{2}-1\right)}{\mathcal{M}^{4}} \varepsilon_{\text {acc }} \quad\left(\varepsilon_{\text {acc }} \ll 1\right) .
$$

Thus, we have $\eta \approx 2.5 \varepsilon_{\text {acc }}$ for $\mathcal{M}=2$ and $\eta \approx 2 \varepsilon_{\text {acc }}$ for $\mathcal{M}=3 .{ }^{13}$ Kang et al. (2007) detect a strong dependence of $\eta$ on the presence of preexisting cosmic rays in the shock upstream, $\varepsilon_{\mathrm{CR}, u}$. The strong dependence can be attributed to inefficient injection at low Mach numbers in their model. For $\mathcal{M}=2$, the parameter $\eta$ jumps from 0 to 0.15 as $\varepsilon_{\mathrm{CR}, u}$ increases from 0 to 0.23 . In stronger

\footnotetext{
12 Once the shock simulation achieves a steady state, $\eta$ approaches the constant ratio between the energy flux in downstream cosmic rays and the bulk kinetic energy flux in the upstream medium entering the shock.

${ }_{13}$ We have here ignored the modification of shock Mach number by the nonthermal pressure; see $\S 2.7$.
}

shocks, for $\mathcal{M}=3$, the parameter $\eta$ jumps from 0.1 to 0.25 for the same increase in $\varepsilon_{\mathrm{CR}, u}$.

Converting our current limits on $\varepsilon_{\text {acc }}$ into limits on $\eta$, we find $\eta \lesssim 0.2$ for $\mathcal{M} \approx 2$ and $\eta \lesssim 0.3$ for $\mathcal{M} \approx 3$. These limits are marginally consistent with the predictions of Kang et al. (2007) for any assumed value of $\varepsilon_{\mathrm{CR}, u}$. However, if as Kang et al. (2007) argue, $\varepsilon_{\text {acc }}$ is itself a sensitive function of $\varepsilon_{\mathrm{CR}, u}$, then improved measurements of $\varepsilon_{\text {acc }}$ that can be obtained with additional observations with existing $\mathrm{X}$-ray telescopes can provide tighter limits on $\varepsilon_{\mathrm{CR}, u}$. Moreover, a positive measurement of $\varepsilon_{\text {acc }}$ can provide a model-dependent constraint on $\varepsilon_{\mathrm{CR}, u}$.

\subsection{X-Ray and SZE Cluster Mass Estimates}

Galaxy cluster surveys in which the cluster masses are measured accurately can be used as powerful cosmological probes of dark matter and dark energy. The mass estimates are plagued by systematic uncertainties that must be understood and quantified before the requisite mass measurement accuracy is achieved. Nonthermal pressure due to cosmic rays, magnetic fields, and turbulence is a source of a systematic bias when cluster masses are estimated on the basis of the assumption of hydrostatic equilibrium between gravitational forces and thermal pressure gradients in the ICM (e.g., Ostriker et al. 2005; Rasia, et al. 2006; Nagai et al. $2007 \mathrm{a}, 2007 \mathrm{~b}$ and references therein). The hydrostatic mass profile of a spherically symmetric cluster is given by

$$
M(<r)=\frac{-r^{2}}{G \rho_{g}}\left(\frac{d P_{g}}{d r}+\frac{d P_{\mathrm{nt}}}{d r}\right),
$$

where $M(<r)$ is the mass enclosed within radius $r$, while $P_{g}$ and $P_{\text {nt }}$ are the thermal and the nonthermal contributions to the pressure. The thermal gas provides a significant fraction of the total pressure support, and this pressure is measured directly with current X-ray and SZE observations. The contribution of the nonthermal pressure, on the other hand, is customarily assumed to be relatively small $(\leqslant 10 \%)$ outside of a cluster core (see e.g., Nagai et al. 2007a, 2007b), and it is often ignored in the hydrostatic mass estimates based on X-ray and SZE data. However, present observations do not yet constrain the nonthermal pressure in the regime in which it dramatically affects the calibration of the hydrostatic mass estimates. If not accounted for, these nonthermal biases will limit the effectiveness of upcoming X-ray and SZE cluster surveys to accurately measure the expansion history of the universe. Detailed investigations of the sources of nonthermal pressure in clusters are thus critical for understanding their effect on the properties of the ICM and the utility of clusters as precision cosmological probes. We proceed to discuss how future observations of cluster merger shocks will have the potential to place unique constraints on the nonthermal pressure in the unshocked ICM, thereby improving cluster mass estimates.

\subsection{Prospects for Future Constraints of Nonthermal Pressure}

As discussed in $\S 3$, current measurements alone do not place strong constraints on the presence of a nonthermal component in the unshocked ICM in both systems. However, the improved constraints that can be obtained with existing X-ray telescopes can provide useful lower limits on nonthermal pressure and their effects on the X-ray and SZE cluster mass estimates. In the case of the shock in the cluster merger A520, the current constraints, which are based on a 67 ks observation with Chandra, can be improved significantly with follow-up observations with Chandra or XMM-Newton. Therefore, we identify this system as the most promising one in which our method may yield a positive shockhydrodynamic detection of a nonthermal component. While the 
stronger shock in the cluster merger 1E 0657-56 may be more efficient at accelerating particles, it will be more difficult to improve the measurement of the shock temperature jump in $1 \mathrm{E}$ 0657-56. This is because the very high temperature of the shock downstream medium $\left(T_{d} \approx 30-50 \mathrm{keV}\right)$ lies far outside of spectral sensitivity window of X-ray telescopes with arcsecond resolution, which in turn renders it difficult to measure the temperature jump in the narrow postshock layer. Hard X-ray observations (e.g., Petrosian et al. 2006) with RXTE, INTEGRAL, or Suzaku, combined with a model of ICM fluid flow (e.g., Milosavljević et al. 2007; Springel \& Farrar 2007), may help to pin down the downstream temperature. An alternative intriguing possibility is that future high-resolution SZE observations may be able to measure the downstream temperature on the basis of the relativistic SZE, which should be prominent in the high temperature downstream.

In the next few years, gamma-ray observations of galaxy clusters may provide tight constraints on the fractional contribution of nonthermal particles to the pressure of the ICM. Assuming that gamma-ray emission from the decay of neutral pions is the primary emission channel, measurements with the new gamma-ray telescope GLAST can be used to place population-averaged limits on the hadronic cosmic-ray pressure support in clusters (Ando \& Nagai 2007; see also Berrington \& Dermer 2003; Blasi et al. 2007). GLAST will also potentially probe the population of accelerated electrons by its contribution the observed extragalactic gamma-ray background (Loeb \& Waxman 2000; Keshet et al. 2004). These forthcoming constraints, combined with improved $\mathrm{X}$-ray spectroscopic measurements of the jump conditions in merger shocks, may enable a separation of the upstream nonthermal pressure into its constituent components.

Finally, comparisons of the hydrostatic mass estimates with those derived from gravitational lensing observations can, in principle, provide an important handle on nonthermal biases. Note that this approach is not practical for individual clusters, because lensing measures the mass in a projected aperture that cannot be directly compared to the mass within a sphere of the same radius, to which the hydrostatic mass is sensitive. But it might be possible to compare different estimators in an average sense, while accounting for the effects of asphericity of clusters and projection effects (see $\S 5.2$ in Nagai et al. 2007b).

\section{CONCLUSIONS}

We model the effect of particle acceleration and nonthermal pressure components on shock jump conditions in nonrelativistic shocks. We focus on intermediate Mach number shocks, with Mach numbers in the range $\mathcal{M}=2-3$. We apply this to the merger shocks in galaxy clusters A520 and 1E 0657-56 and place the first constraints on the efficiency of particle acceleration in these shocks. Our main results are as follows.

1. The temperature jump of the thermal gas in the shock depends strongly on the efficiency of shock particle acceleration. Efficient acceleration can reduce the temperature jump by more than a factor of 2 for a constant compression ratio in the range $r=2-3$.

2. The correct effect of nonthermal pressure in the upstream, such as fossil cosmic rays, magnetic field, and turbulence, on the shock jump observed in the thermal gas cannot be derived at this point, because we lack an understanding of the interaction of these components with the shock. However, for a wide range of reasonable assumptions and analytic approximations, we find that nonthermal pressure in the unshocked ICM has only a minor effect on the downstream temperature (at a fixed compression ratio), and that in general, a high upstream nonthermal pressure increases the temperature jump in the thermal gas.

3 . The combination of a strong dependence of the temperature jump on particle acceleration and a weak dependence on upstream nonthermal pressure enables derivation of meaningful constraints on the efficiency of particle acceleration in cluster merger shocks, even with current observations. Future, more accurate X-ray and SZE observations of these shocks may yield meaningful constraints on the upstream nonthermal pressure as well.

4. Nonthermal pressure and shock particle acceleration can also affect the Mach number that is inferred from the observed compression ratio $r$ by tens of percent. When the temperature jump is poorly constrained, the Mach number is anticorrelated with efficient particle acceleration and is positively correlated with upstream nonthermal pressure.

5. In the two observed high-contrast galaxy cluster merger shocks, A520 and 1E 0657-56, we constrain the acceleration efficiency of relativistic particles to be $\varepsilon_{\text {acc }} \lesssim 0.1$ and $\varepsilon_{\text {acc }} \lesssim 0.15$, respectively. We find that considerable upstream pressure can increase the Mach number of the shock in A520 to reach $\mathcal{M} \approx 2.7$, much higher than the inferred value of 2.1 obtained assuming an absence of nonthermal components. The true Mach number of the shock in 1E $0657-56$ can be in the range $2.3<\mathcal{M}<3.7$ with the compression ratio of $r=3$, allowing for nonthermal pressure components.

The research was supported in part by the Sherman Fairchild Foundation. We would like to thank A. Königl, E. Komatsu, A. Kravtsov, P. Kumar, Y. Rephaeli, and J. Scalo for helpful discussions.
Achterberg, A., Blandford, R., \& Periwal, V. 1984, A\&A, 132, 97

Aharonian, F., et al. 2007, A\&A, 464, 235

Ando, S., \& Nagai, D. 2007, preprint (arXiv/0705.2588)

Andreopoulos, Y., Agui, J. H., \& Briassulis, G. 2000, Annu. Rev. Fluid Mech., 32, 309

Baring, M. G., Ogilvie, K. W., Ellison, D. C., \& Forsyth, R. J. 1997, ApJ, 476, 889

Batchelor, G. K. 1953, The Theory of Homogeneous Turbulence (Cambridge: Cambridge Univ. Press)

Bell, A. R. 1978, MNRAS, 182, 147

2004, MNRAS, 353, 550

2005, MNRAS, 358, 181

Bell, A. R., \& Lucek, S. G. 2001, MNRAS, 321, 433

Berezinsky, V. S., Blasi, P., \& Ptuskin, V. S. 1997, ApJ, 487, 529

Berrington, R. C., \& Dermer, C. D. 2003, ApJ, 594, 709

Blandford, R., \& Eichler, D. 1987, Phys. Rep., 154, 1

Blandford, R. D., \& Ostriker, J. P. 1978, ApJ, 221, L29

Blasi, P., Gabici, S., \& Brunetti, G. 2007, Int. J. Mod. Phys., A22, 681

\section{REFERENCES}

Carilli, C. L., \& Taylor, G. B. 2002, ARA\&A, 40, 319

Dolag, K., Vazza, F., Brunetti, G., \& Tormen, G. 2005, MNRAS, 364, 753

Drury, L. O., \& Völk, J. H. 1981, ApJ, 248, 344

Ellison, D. C., Moebius, E., \& Paschmann, G. 1990, ApJ, 352, 376

Enßlin, T. A., Biermann, P. L, Kronberg, P. P., \& Wu, X.-P. 1997, ApJ, 477, 560

Faltenbacher, A., Kravtsov, A. V., Nagai, D., \& Gottlöber, S. 2005, MNRAS, 358,139

Farrar, G. R., \& Rosen, R. A. 2007, Phys. Rev. LeH., 98, 171302

Fujita, Y., \& Sarazin, C. L. 2001, ApJ, 563, 660

Fujita, Y., Matsumoto, T., \& Wada, K. 2004, ApJ, 612, L9

Gabici, S., \& Blasi, P. 2003, ApJ, 583, 695

Giacalone, J., Burgess, D., Schwartz, S. J., Ellison, D. C., \& Bennett, L. 1997, J. Geophys. Res., 102, 19789

Govoni, F., \& Feretti, L. 2004, Int. J. Mod. Phys. D, 13, 1549

Govoni, F., Murgia, M., Feretti, L., Giovannini, G., Dolag, K., \& Taylor, G. B.

2006, A\&A, 460, 425

Hayashi, E., \& White, S. D. M. 2006, MNRAS, 370, L38

Inogamov, N. A., \& Sunyaev, R. A. 2003, Astron. Lett., 29, 791 
Jacquin, L., Cambon, C., \& Blin, E. 1993, Phys. Fluids, 5, 2539

Kang, H., \& Jones, T. W. 2007, Astropart. Phys., 28, 232

Kang, H., Jones, T. W., \& Gieseler, U. D. J. 2002, ApJ, 579, 337

Kang, H., Ryu, D., Cen, R., \& Ostriker, J. P. 2007, ApJ, 669, 729

Keshet, U., Waxman, E., \& Loeb, A. 2004, J. Cosmol. Astropart. Phys., 4, 6

Konigl, A. 1980, Phys. Fluids, 23, 1083

Kunik, M., Qamar, S., \& Warnecke, G. 2003, J. Comput. Phys., 187, 572

Lee, S., Lele, S. K., \& Moin, P. 1993, J. Fluid Mech., 251, 533 1997, J. Fluid Mech., 340, 225

Lele, S. K. 1992, Phys. Fluids, 4, 2900

Loeb, A., \& Waxman, E. 2000, Nature, 405, 156

Lucek, S. G., \& Bell, A. R. 2000, MNRAS, 314, 65

Malkov, M. A., \& O'C Drury, L. 2001, Rep. Prog. Phys., 64, 429

Markevitch, M. 2006, The X-ray Universe 2005 (ESA SP-604; Garching: ESA), 723

Markevitch, M., Gonzalez, A. H., David, L., Vikhlinin, A., Murray, S., Forman, W., Jones, C., \& Tucker, W. 2002, ApJ, 567, L27

Markevitch, M., Govoni, F., Brunetti, G., \& Jerius, D. 2005, ApJ, 627, 733

Markevitch, M., \& Vikhlinin, A. 2007, Phys. Rep., 443, 1

Medvedev, M. V., Silva, L. O., \& Kamionkowski, M. 2006, ApJ, 642, L1

Milosavljević, M., Koda, J., Nagai, D., Nakar, E., \& Shapiro, P. R. 2007, ApJ, 661, L131

Miniati, F., Ryu, D., Kang, H., \& Jones, T. W. 2001, ApJ, 559, 59

Nagai, D., Kravtsov, A. V., \& Kosowsky, A. 2003, ApJ, 587, 524

Nagai, D., Kravtsov, A. V., \& Vikhlinin, A. 2007a, ApJ, 668, 1

Nagai, D., Vikhlinin, A., \& Kravtsov, A. V. 2007b, ApJ, 655, 98
Norman, M. L., \& Bryan, G. L. 1999, The Radio Galaxy Messier 87, ed. H.-J. Röser \& K. Meisenheimer (Berlin: Springer), 106

Ostriker, J. P., Bode, P., \& Babul, A. 2005, ApJ, 634, 964

Petrosian, V., Madejski, G., \& Luli, K. 2006, ApJ, 652, 948

Pfrommer, C., \& Enßlin, T. A. 2004, A\&A, 413, 17

Rasia, E., et al. 2006, MNRAS, 369, 2013

Reimer, O., Pohl, M., Sreekumar, P., \& Mattox, J. R. 2003, ApJ, 588, 155

Ricker, P. M., \& Sarazin, C. L. 2001, ApJ, 561, 621

Rotman, D. 1991, Phys. Fluids, 3, 1792

Ryu, D., Kang, H., Hallman, E., \& Jones, T. W. 2003, ApJ, 593, 599

Sarazin, C. L. 2004, J. Korean Astron. Soc., 37, 433

Schekochihin, A. A., Cowley, S. C., Kulsrud, R. M., Hammett, G. W., \& Sharma, P. 2005, ApJ, 629, 139

Schlickeiser, R., \& Shukla, P. K. 2003, ApJ, 599, L57

Schuecker, P., Finoguenov, A., Miniati, F., Böhringer, H., \& Briel, U. G. 2004, A\&A, 426, 387

Springel, V., \& Farrar, G. 2007, MNRAS, 380, 911

Subramanian, K., Shukurov, A., \& Haugen, N. E. L. 2006, MNRAS, 366, 1437

Sunyaev, R. A., Norman, M. L., \& Bryan, G. L. 2003, Astron. Lett., 29, 783

Vazza, F., Tormen, G., Cassano, R., Brunetti, G., \& Dolag, K. 2006, MNRAS, 369, L14

Vikhlinin, A., Markevitch, M., \& Murray, S. S. 2001, ApJ, 549, L47

Warren, J. S., et al. 2005, ApJ, 634, 376

Zank, G. P., Zhou, Y., Matthaeus, W. H., \& Rice, W. K. M. 2002, Phys. Fluids, 14,3766 